\title{
Amyloid Precursor Protein Overexpression in Down Syndrome Trophoblast Reduces Cell Invasiveness and Interferes with Syncytialization
}

\author{
Oscar G.W. Wong, ${ }^{*}$ Claire L.Y. Cheung, ${ }^{*}$ Philip P.C. Ip, ${ }^{*}$ Hextan Y.S. Ngan, ${ }^{\dagger}$ and Annie N.Y. Cheung *
}

From the Departments of Pathology* and Obstetrics and Gynaecology, ${ }^{\dagger}$ The University of Hong Kong, Hong Kong, People's Republic of China

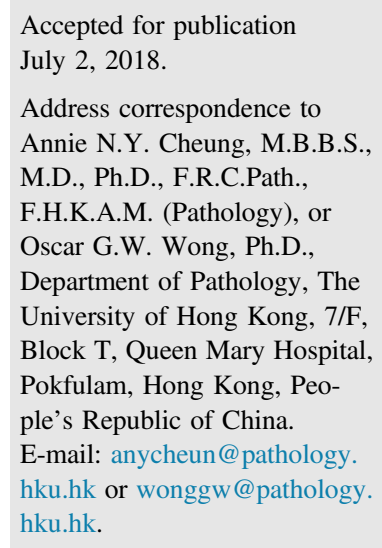

\begin{abstract}
The placentas of Down syndrome (DS) pregnancies exhibit morphologic and functional abnormalities. Although the increase in dosage of certain genes on chromosome 21 has been associated with the DS phenotype, the effects on placenta have seldom been studied. Herein, we examine the expression of four dosage-sensitive genes (APP, ETS2, SOD1, and HMGN1) in normal and DS placentas. We demonstrated significant overexpression of amyloid precursor protein (APP) in DS placentas at RNA and protein levels by real-time quantitative PCR, Western blot analysis, and immunohistochemistry. Inducible APP overexpression trophoblast cell line models were established using a Tet-0n system. APP induction in HTR-8/ SVneo dose-dependently decelerated cell growth, enhanced apoptosis, and reduced cell migration and invasion when compared with the uninduced controls. Concomitantly, decreased $\beta$-human chorionic gonadotropin in the culture medium was also detected on induction. Moreover, although forskolin treatment induced $\alpha / \beta$-human chorionic gonadotropin and syncytin expression in BeWo cells, such induction of syncytialization was inhibited by APP overexpression. E-cadherin immunofluorescence also demonstrated a decrease in syncytia formation in forskolin-treated BeWo-overexpressing APP. By liquid chromatography-tandem mass spectrometry, proteins related to cell-cell adhesion, protein translation, processing, and folding were found to be up-regulated in APP-induced HTR-8/SVneo clones. Our data demonstrated, for the first time, the effects of increased APP expression in DS placenta. (Am J Pathol 2018, 188: 2307-2317; https://doi.org/10.1016/j.ajpath.2018.07.004)
\end{abstract}

Down syndrome (DS) is the most common congenital abnormality in humans, affecting 1 in approximately 1000 live births worldwide. An extra chromosome 21 (trisomy 21 ) is the major cause, accounting for approximately $95 \%$ of all cases. DS manifests in a spectrum of phenotypes, including challenges in cognitive ability and physical growth, cardiac defects, craniofacial alterations, and muscle hypotonia. ${ }^{1}$ Growth retardation in trisomy 21 fetuses is partly attributable to placental structural abnormalities. ${ }^{2,3}$

Various developmental defects in the placentas of DS pregnancies have been reported. ${ }^{4}$ The chorionic villi in trisomy 21 were reported to be bigger and more asymmetric and irregular in shape than were normal villi. ${ }^{2,5}$ Histomorphometrically, the villi in trisomy 21 placentas had an increased percentage of two-layered trophoblast and an increased proportion of villus capillaries with nucleated red blood cells. ${ }^{6}$ The increased two-layered trophoblast phenotype of trisomy 21 placenta has been postulated to be the consequence of defective trophoblast fusion and differentiation, ${ }^{4}$ because it has been shown that cultured villous cytotrophoblast cells from placentas of trisomy 21 fused inefficiently to form multinucleated syncytiotrophoblast. ${ }^{7}$ The inefficient differentiation from cytotrophoblast into syncytiotrophoblast leads to the secretion of an abnormal and weakly bioactive human chorionic gonadotropin (hCG) and results in defective regulation of placental

\footnotetext{
Supported by the Health and Medical Research Fund, Food and Health Bureau, Hong Kong Special Administrative Region project number 01120936 (A.N.Y.C.).

Disclosures: None declared.
} 
development. ${ }^{8}$ In addition, the number of mature hCG receptor (LHCGR) molecules expressed on the surface of trisomy 21-affected cytotrophoblasts has also been shown to reduce significantly compared with normal cytotrophoblasts. ${ }^{9}$ The molecular basis underlies these alterations in trophoblast differentiation, and hCG signaling in DS placentas is largely unknown.

It is believed that the various DS phenotypes are the results of the extra copy of dosage-sensitive genes among the genes present on human chromosome 21. A list of genes with direct evidence that their increased dose or allelic variation induces one or more phenotypes of DS has been compiled. ${ }^{10}$ Microarray analysis of genes dysregulated in cultured trisomy 21 trophoblasts also revealed highly up-regulated genes on chromosome 21 in DS trophoblasts, supporting the hypothesis that candidate dosage-sensitive genes are involved in DS pathogenesis. ${ }^{11}$

The gene APP encoding amyloid precursor protein (APP) is located on chromosome 21 and is one of the earliest genes known to be overexpressed in the brain of DS patients. ${ }^{12}$ Pathologic cleavage of APP into amyloid- $\beta$ protein is a hallmark of Alzheimer disease. Overexpression of APP in DS brain contributes to the high penetrance of early-onset Alzheimer disease commonly seen in DS patients. ${ }^{13} \mathrm{Up}$ regulation of APP in other organs/tissues of DS patients has also been reported. ${ }^{14-16}$ It was also one of the most significantly overexpressed genes in cultured trisomy 21 trophoblasts. ${ }^{11}$ However, whether APP is contributing to the DS placental phenotypes is unclear.

In this study, we explored the expression patterns and functional impact of one of the four previously suggested dosagesensitive genes (APP, ETS2, SODI, and HMGN1) in DS placentas. ${ }^{10}$ APP was found to be significantly overexpressed in DS placentas. An in vitro inducible expression system was then established to study the functional impact of APP overexpression on trophoblast biology. Our results suggest that APP is contributing to the defective trophoblast phenotype of DS.

\section{Materials and Methods}

\section{Clinical Samples}

Seventy-one formalin-fixed, paraffin-embedded (FFPE) placental tissues, including 37 placentas from normal pregnancies (4 first trimester, 31 second trimester, and 2 third trimester) and 34 placentas from DS pregnancies (2 first trimester, 30 second trimester, 2 third trimester) were retrieved from the archive of the Department of Pathology, Queen Mary Hospital (Pokfulam, Hong Kong). Each block was confirmed to contain only placental tissue by hematoxylin and eosin staining. The use of the clinical samples for this study has been reviewed and approved by the HKU/Hospital Authority Hong Kong West Cluster Institutional Review Board (reference UW 13-124).

\section{Cell Lines}

The trophoblast cell line HTR-8/SVneo was a gift from Dr. Peeyush K. Lala (University of Western Ontario, London,
Canada) ${ }^{17}$ and was cultured in RPMI 1640 medium supplemented with $10 \%$ heat-inactivated qualified fetal bovine serum (catalog number 16140071; Gibco, Waltham, MA) and $0.01 \%$ penicillin-streptomycin. The choriocarcinoma cell line, BeWo, was purchased from ATCC (Manassas, VA) and was cultured in F-12K supplemented with $10 \%$ heat-inactivated qualified fetal bovine serum and $0.01 \%$ penicillin-streptomycin. The cells were maintained at $37^{\circ} \mathrm{C}$ under $5 \% \mathrm{CO}_{2}$.

\section{RNA Extraction and RT-qPCR}

Five sections ( $10 \mu \mathrm{m}$ thick) were cut from each placenta FFPE block, and the total RNA was extracted using RNeasy FFPE kit (Qiagen, Venlo, the Netherlands). The quality of the samples was analyzed by a Bioanalyzer (Agilent, Santa Clara, CA). The mean RNA integrity number was 2.497 $(\mathrm{SD}=0.9592$; mean RNA integrity number normal $=2.495$; mean RNA integrity number DS $=2.500$ ). RNA of such quality is considered acceptable for RNA extracted from FFPE tissue and has been used in previous gene expression studies using real-time quantitative RT-PCR (RT-qPCR). ${ }^{18}$ At least $500 \mathrm{ng}$ total RNA was reverse transcribed into cDNA using a PrimeScript RT kit (Clontech, Mountain View, $\mathrm{CA}$ ), according to the manufacturer's protocol. Each RT-qPCR contains $1 \times$ HotStart SYBR Green qPCR master mix (Excell, Shanghai, China), $1 \mu \mathrm{mol} / \mathrm{L}$ forward primer, $1 \mu \mathrm{mol} / \mathrm{L}$ reverse primer, $0.5 \mu \mathrm{L}$ cDNA, and $4.5 \mu \mathrm{L}$ milli-Q water. RT-qPCRs were run using a 7900HT Fast Real-Time PCR System (Applied Biosystems, Foster City, CA). Sequences of the primers used were as follows: $A P P, 5^{\prime}$ ATCTCCGCGGGGATCCATGCTGCCCGGTTTGGCACTGCTC- $3^{\prime}$ (forward) and 5'-GCGGATCGATGGATCCCTAGTTCTGCATCTGCTCAAAGAA-3' (reverse); ETS2, 5'-ATCTCCGCGGGGATCCATGAATGATTTCGGAATCAAGAAT- $3^{\prime}$ (forward) and $5^{\prime}$-GCGGATCGATGGATCCTCAGTCCTCCGTGTCGGGCTGGAC-3' (reverse); SODI, 5'-ATCTCCGCGGGGATCCATGGCGACGAAGGCCGTGTGCGTG-3' (forward) and 5'-GCGGATCGATGGATCCTTATTGGGCGATCCCAATTACACC-3' (reverse); $H M G N 1,5^{\prime}$-ATCTCCGCGGGGATCCATGCCCAAGAGGAAGGTCAGCTCC-3' (forward) and $5^{\prime}$-GCGGATCGATGGATCCTTAATCAGACTTGGCTTCTTTCTC-3' (reverse); YWHAZ, 5'-ACTTTTGGTACATTGTGGCTTCAA-3' (forward) and 5'-CCGCCAGGACAAACCAGTAT- $3^{\prime}$ (reverse); glyceraldehyde-3-phosphate dehydrogenase, 5'-AGATCATCAGCAATGCCTCC- $3^{\prime}$ (forward) and 5'-CATGAGTCCTCCCACGATAC-3' (reverse); and TOP1, 5'-GATGAACCTGAAGATGATGGC-3' (forward) and $5^{\prime}$-TCAGCATCATCCTCATCTCG-3' (reverse).

\section{Immunohistochemistry}

The method of choice for antigen retrieval in immunohistochemistry was by heating the FFPE sections bathed in TE buffer $(10 \mathrm{mmol} / \mathrm{L}$ Tris, $\mathrm{pH} 8.0$, and $5 \mathrm{mmol} / \mathrm{L}$ EDTA $)$ in a pressure cooker. The sections were then incubated with 
anti-APP antibody (catalog number ab32136; Abcam, Cambridge, UK) (dilution, 1:250) at $4^{\circ} \mathrm{C}$ overnight, and the signal was visualized using the EnVision+ Dual Link System (Agilent, Santa Clara, CA). ${ }^{19}$ Negative control was prepared by replacing the primary antibody with phosphatebuffered saline. ${ }^{20}$ Fetal and placental tissue found to be positive was used as positive control. The stained sections were scanned with an Aperio slide scanner (Leica Biosystems, Wetzlar, Germany). The representative regions of trophoblast on each section were marked by a pathologist (A.N.Y.C.), and the staining intensity of the regions was evaluated by the positive pixel count $\mathrm{v} 9$ algorithm included in the system.

\section{Western Blot Analysis}

Western blot analysis was performed according to standard procedures. Antibodies used include the following: APP (1:1000; catalog number ab32136; Abcam), green fluorescent protein (1:1000; ZsGreen; catalog number 632598; Clontech), $\beta$-actin $(1: 10,000$; catalog number A5060; Sigma-Aldrich, St. Louis, MO), caspase 3 (1:1000; catalog number 9662; Cell Signaling, Danvers, MA), phosphorylated extracellular signal-regulated kinase (ERK) $1 / 2$ (1:1000; catalog number 4376; Cell Signaling), ERK1/2 (1:3000; catalog number 9102; Cell Signaling), and LHCGR (1:1000; catalog number 19968-1-AP; Proteintech, Rosemont, IL).

\section{Establishment of the Inducible Overexpression Trophoblast Cell Line Models}

The functional implication of APP overexpression was investigated in a previously established trophoblast cell line HTR-8/SVneo. ${ }^{17}$ HTR-8/SVneo was stably transfected with a Tet-On expression plasmid harboring APP ORF pTRE3GBI-APP. Two clones, B2 and B10, were selected. APP was induced in $\mathrm{B} 2$ and $\mathrm{B} 10$ by including the recombinant protein Tet-Express (Clontech) in the culture medium.

For assessing the impact of APP overexpression on syncytialization, BeWo was first stably transfected with pCMV-TET3G, which expresses the Tet-On 3G transactivator protein. BeWo-tet3G was then transiently transfected with pTRE3G-BI-APP using PolyJet (Signagen, Rockville, MD), according to the manufacturer's protocol, and APP was induced with doxycycline.

\section{Cell Functional Assays}

Growth of induced cells over a period of 6 days was evaluated and compared using MTT assay, according to standard protocol. The rates of cell proliferation of induced cells were evaluated using bromodeoxyuridine (BrdU) assay 2 days after induction (Cell Signaling), according to the protocol provided by the manufacturer. DNA fragmentation was measured by flow cytometry analysis of propidium iodide stained cells for sub-G1 fraction. Apoptotic cells in cultured cells and FFPE tissue sections were visualized by TdT-mediated dUTP nick-end labeling (TUNEL) assay using the DeadEnd Colorimetric TUNEL System (Promega, Madison, WI), according to the protocol provided by the manufacturer. Cell mobility and invasiveness were evaluated using transwell migration/invasion assays, as previously described. ${ }^{19}$ Briefly, induced B2 and B10 cells were seeded into the upper chamber of transwell inserts at a density of $3 \times 10^{5}$ per insert. The inserts were uncoated for migration assay or coated with Matrigel (Corning Life Sciences, Oneonta, NY) for invasion assay. The cells were allowed to migrate through the membrane for 24 hours, after which the cells in the upper chamber were removed and migrated cells were stained with crystal violet. Syncytialization of BeWo treated with $100 \mu \mathrm{mol} / \mathrm{L}$ forskolin was visualized by immunofluorescence staining of E-cadherin (catalog number 610181; BD Biosciences, San Jose, CA). Syncytialization was quantified by dividing the number of nuclei belonging to fused cells by the total number of nuclei in a field. Five fields were counted for each condition.

\section{hCG Assay}

The concentration of total $\beta$-hCG in the conditioned medium of B2 and B10 with or without induction by TetExpress was measured by ARCHITECT total $\beta$-hCG test (Abbott Ireland, Longford, Ireland), according to the manufacturer's instruction.

\section{Liquid Chromatography-Tandem Mass Spectrometry}

The sample processing and liquid chromatography-tandem mass spectrometry was performed in the $\mathrm{Li}$ Ka Shing Faculty of Medicine, Proteomics and Metabolomics Core Facility, The University of Hong Kong. Proteins were extracted from cell lysates of uninduced and induced (with $4 \mathrm{ng} / \mu \mathrm{L}$ Tet-Express protein) HTR-8/ SVneo-APP by bead beating using Precellys homogenizer (Bertin Technologies, Versailles, France). Three replicates of each protein sample were subjected to trypsin digestion, followed by reduction and alkylation using a filter-aided sample preparation method. ${ }^{21}$ LysC-Tryptic peptides were extracted, cleaned, and reconstituted for liquid chromatography-tandem mass spectrometry analysis performed on an Orbitrap Fusion Lumos mass spectrometer (Thermo Fisher Scientific, Bremen, Germany) interfaced with a Dionex 3000RSLC nanoLC (Thermo Fisher Scientific, San Jose, CA). The high-resolution, high-mass accuracy mass spectrometry data obtained were processed using Maxquant version 1.5.3.30 (Max Planck Institute of Biochemistry, Munich, Germany), wherein data were searched using the Andromeda algorithm against National Center for Biotechnology Information human RefSeq protein database. Appropriate parameters were used to obtain data at $0.1 \%$ false discovery rate at peptide and protein 
levels. Proteins identified from both uninduced and induced conditions were quantified using the peptide label-free quantification intensities, and their ratio obtained was used for label-free quantitation to calculate the fold change. Data visualization and statistical data analysis were performed with Perseus software version 1.5.4.1 (Max Planck Institute of Biochemistry).

\section{Results}

APP Is Overexpressed in the Placentas of DS Pregnancies

To investigate the expression of the four candidate dosagesensitive genes (APP, ETS2, SOD1, and HMGN1) in DS placentas, RT-qPCR was used to measure the relative expression levels of the genes in FFPE samples of placentas from normal and DS pregnancies. YWHAZ, which was previously shown to be a suitable reference gene for gene expression studies in placenta, was used as the reference gene. $^{22}$ Two additional reference genes (GAPDH and $T O P 1)$ were used for further validation and yielded similar observations. $^{23}$

Although all four genes are located on chromosome 21, not all of them are overexpressed in DS placenta. APP mRNA was, on average, overexpressed by approximately 2.3-fold in DS placenta than in normal placentas $(P<0.0009)$ (Figure 1A). SODl was slightly overexpressed, but did not reach statistical significance $(P=0.3155)$ (Figure 1A). ETS2 and HMGN1, on the other
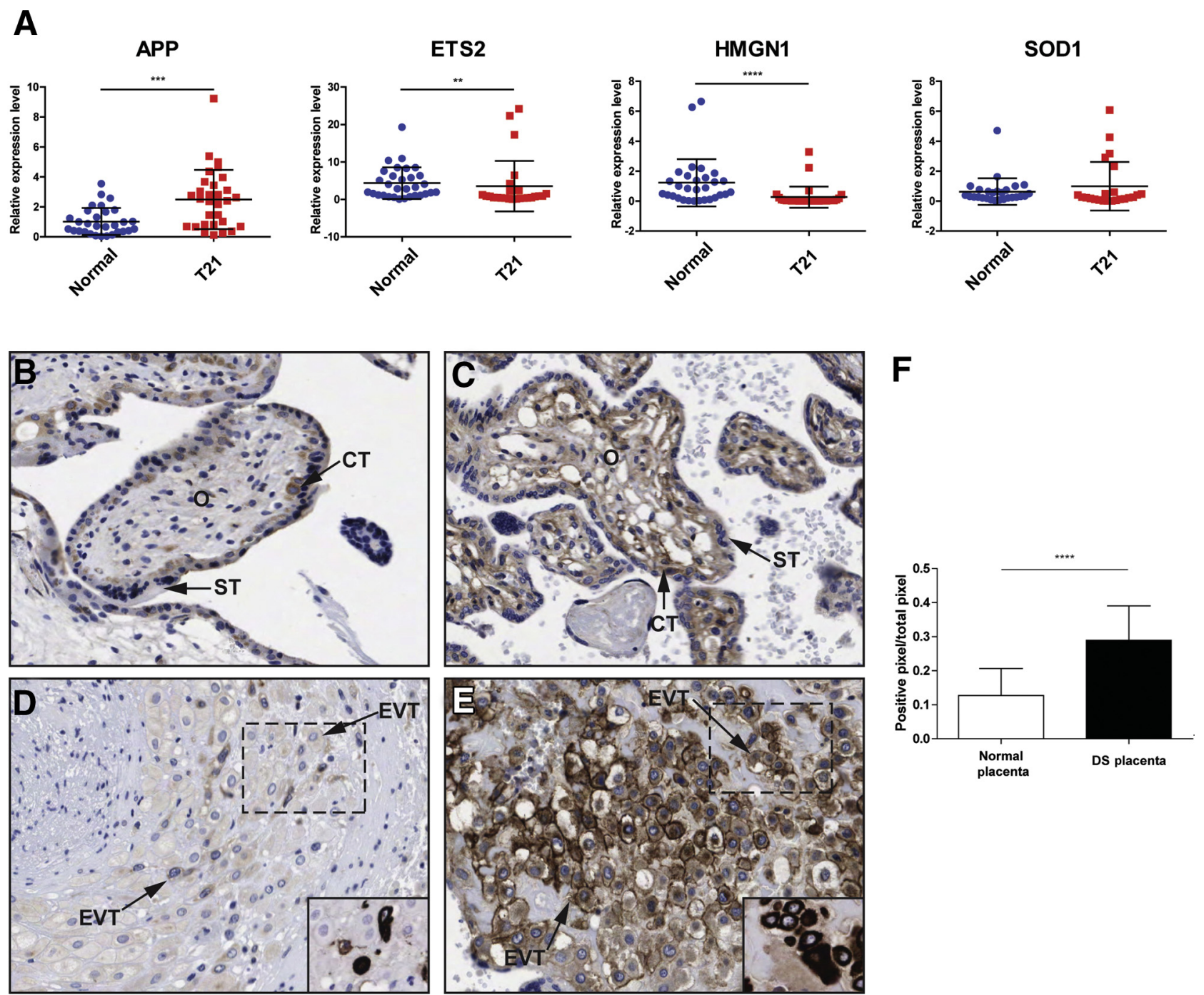

Figure 1 Amyloid precursor protein (APP) is overexpressed in Down syndrome (DS) placenta. A: Expression of APP, ETS2, HMGN1, and S0D1 mRNA was measured in placenta samples by real-time quantitative RT-PCR. B-E: Immunohistochemical study of APP in chorionic villi (B and C) and extravillous trophoblasts ( $\mathbf{D}$ and $\mathbf{E}$ ) of a 21-week normal placenta (B and $\mathbf{D}$ ) and a 23-week DS placenta (C and $\mathbf{E})$. Insets in $\mathbf{D}$ and $\mathbf{E}$ : Immunoreactivity for cytokeratin 7 at the boxed area marked by the dashed line at an adjacent section. F: APP immunoreactivity was quantified by pixel counting using Aperio. ${ }^{* *} P<0.01$, ${ }^{* * *} P<0.001$, and $* * * P<0.0001$. Original magnification, $\times 20$ (B-E). CT, cytotrophoblast; EVT, extravillous intermediate trophoblast; 0 , villi stroma; ST, syncytiotrophoblast; T21, trisomy 21. 
A

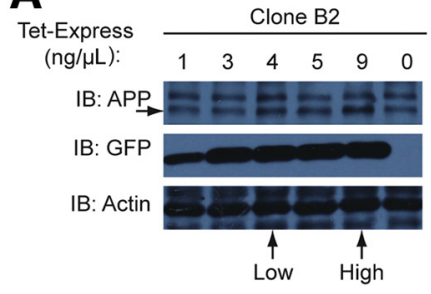

C
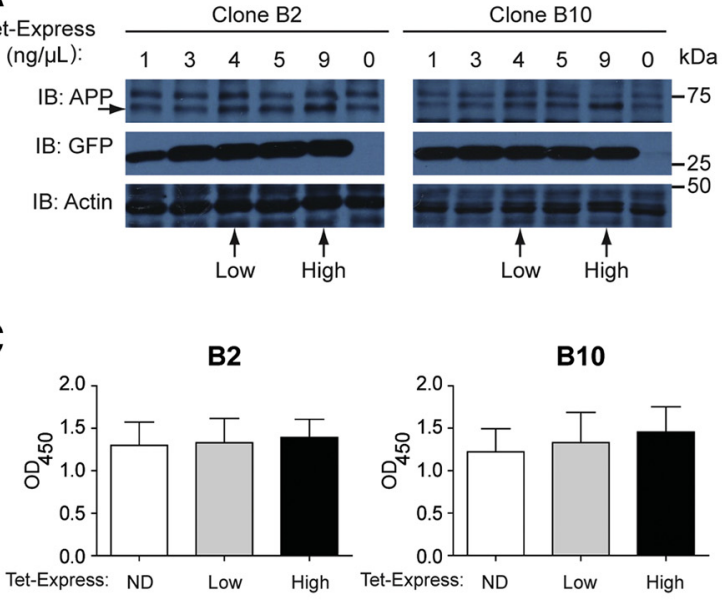

B

\section{F}
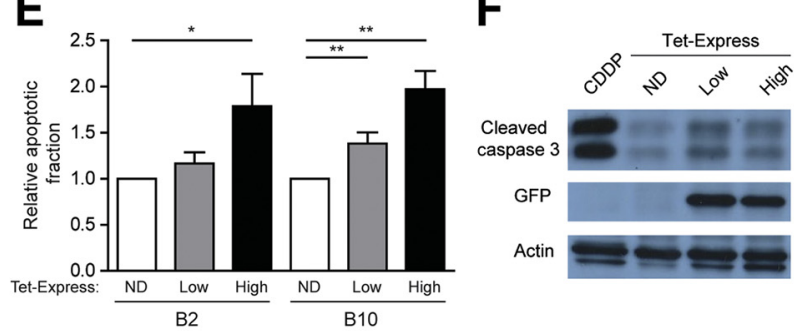

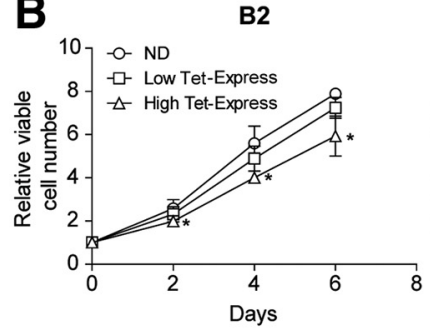

D

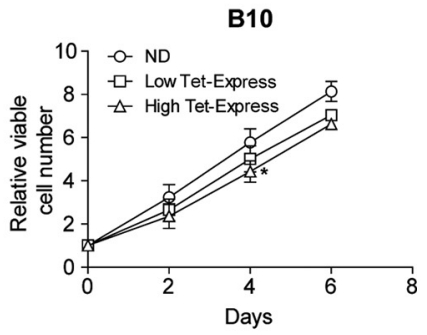

B2
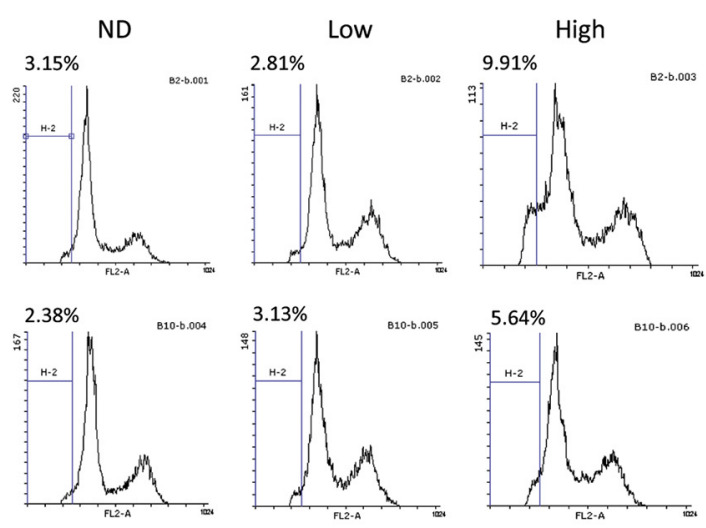

G

ND

Low

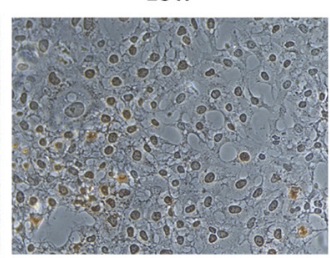

Low
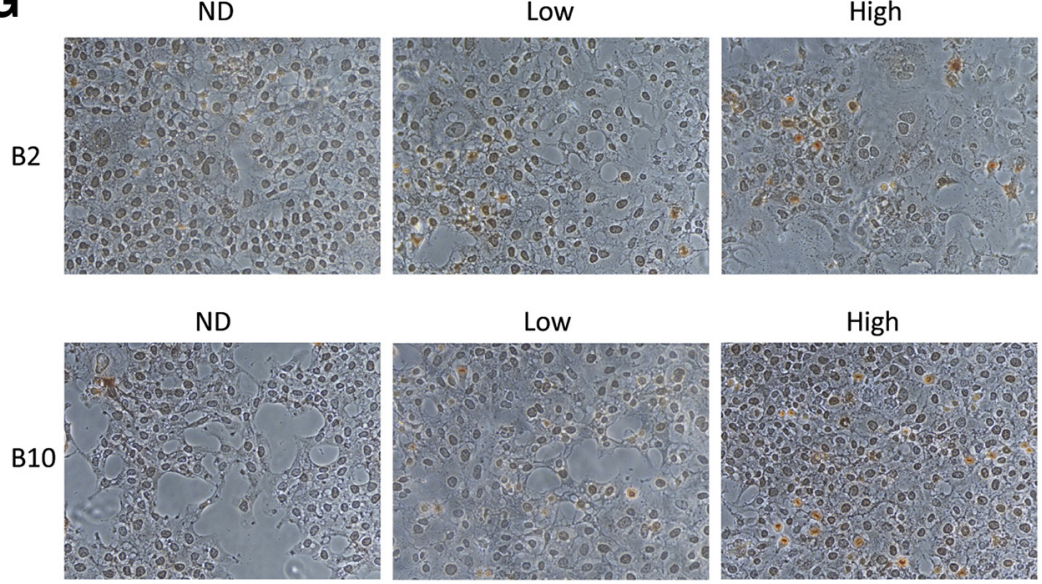

High
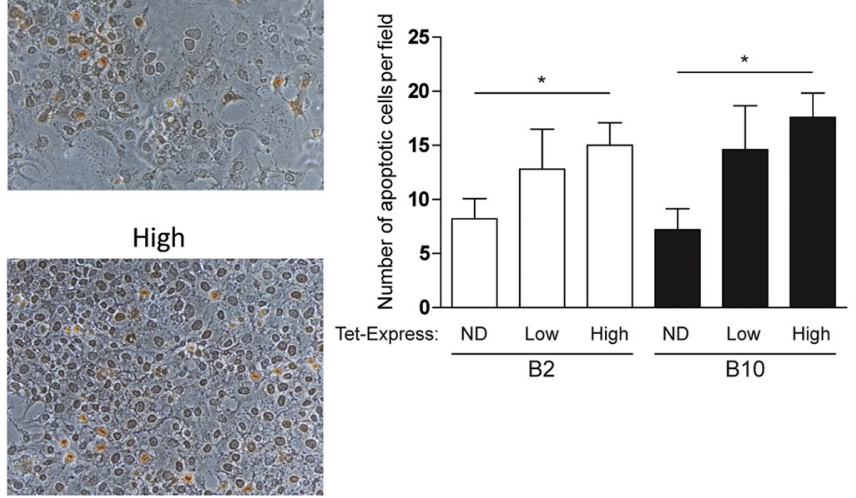

H

Normal

DS
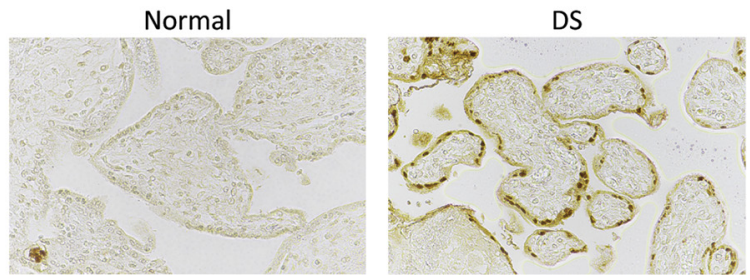

Figure 2 Induced amyloid precursor protein (APP) overexpression suppresses trophoblast cell growth. A: HTR-8/SVneo was stably transfected with pTRE3GBI-APP, which express APP and ZsGreen [green fluorescent protein (GFP)] simultaneously on induction by Tet-Express. B: B2 and B10 uninduced (ND) or induced with the indicated dose of Tet-Express were monitored for their cell growth by MTT assay. C: Induced B2 and B10 were compared with uninduced cells for bromodeoxyuridine (BrdU) incorporation after 24 hours of induction. D: Induced cells were analyzed for DNA fragmentation by propidium iodide staining, followed by flow cytometry analysis. The sub-G1 population was quantitated and presented as percentage of total counted cells. E: Mean percentage sub-G1 population of induced cells normalized to that of uninduced cells in seven independent experiments. F: Caspase-3 cleavage was monitored in induced cells by Western blot. G: Apoptotic cell death in induced cells was examined with TdT-mediated dUTP nick-end labeling (TUNEL) assay. Right panel shows mean number of apoptic cells per field from two independent experiments. Five fields per slide were counted in each experiment. H: Apoptotic cell death in normal and Down syndrome (DS) placenta chorionic villi was examined by TUNEL assay. Data are expressed as means \pm SD (B) or means \pm SEM (E and $\mathbf{G}$ ). $n=4$ independent experiments (B). ${ }^{*} P<0.05,{ }^{* *} P<0.01$. Original magnification: $\times 40(\mathbf{G}) ; \times 20(\mathbf{H})$. CDDP, cisplatin; IB, immunoblot. 
A

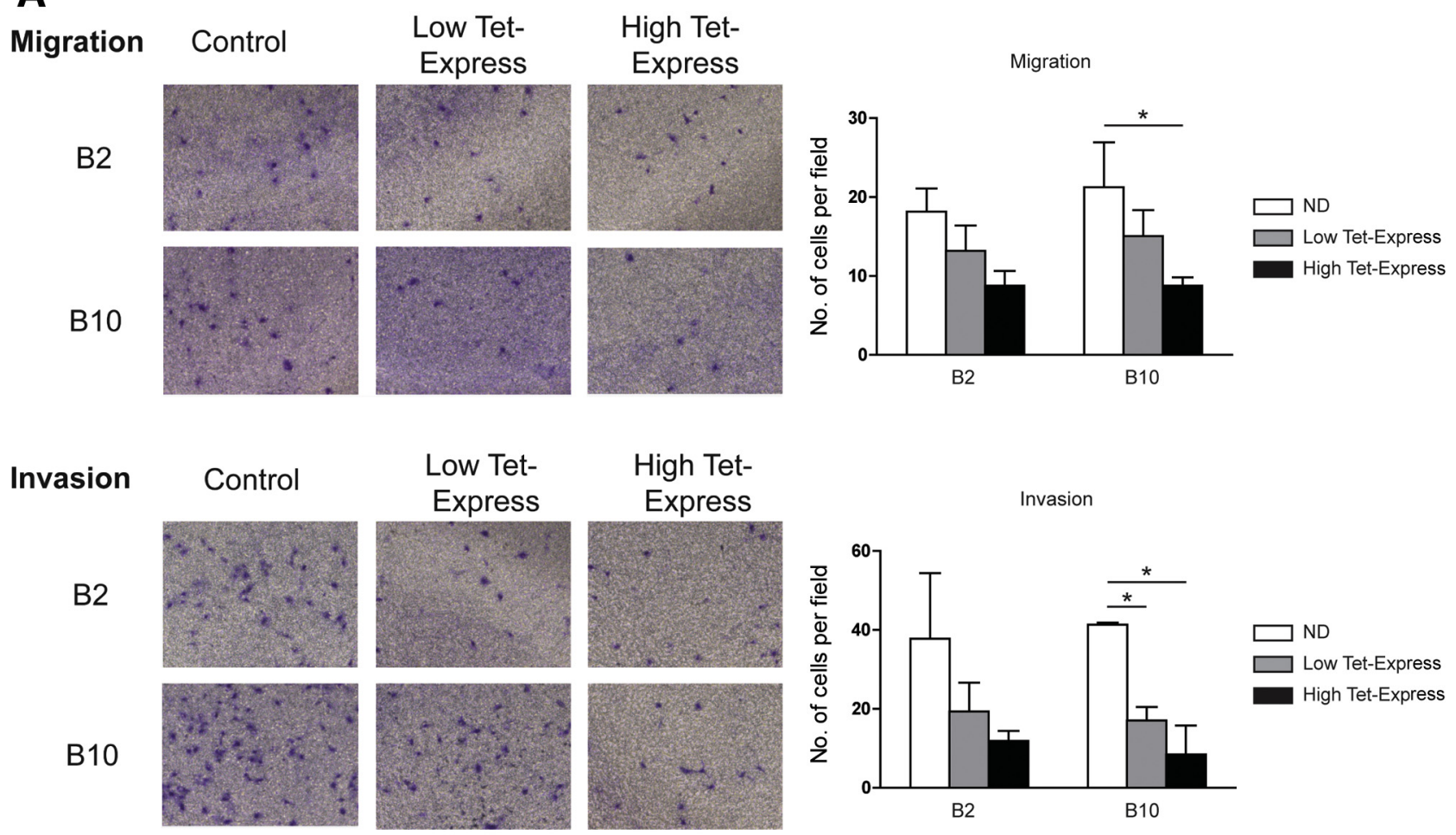

B

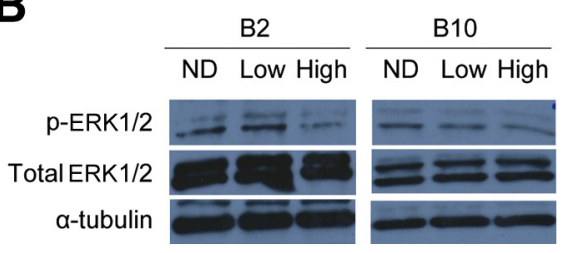

Figure 3 Amyloid precursor protein (APP) overexpression impedes trophoblast migration/ invasion. A: HTR-8/SVneo-APP clones were induced to express APP and allowed to migrate through transwells for 24 hours. For invasion assay, the transwells were coated with Matrigel. B: Phosphorylation of extracellular signal-regulated kinase 1/2 (p-ERK1/2), a transcription factor regulating trophoblast mobility and invasiveness, is down-regulated in cells induced to express APP. Cell lysate was collected 2 days after induction. Data are expressed as means $\pm S D$ from at least three independent experiments (A, right panels). ${ }^{\star} P<0.05$. Original magnification, $\times 10(A)$. ND, uninduced. hand, were both statistically significantly down-regulated (Figure 1A). Because APP was shown to be overexpressed in DS placenta, it was chosen for further investigation.

To characterize the spatial expression pattern of $A P P$ in placentas, the APP protein was stained in the FFPE tissue samples by immunohistochemistry. Cytoplasmic immunoreactivity for APP was found predominantly in villous cytotrophoblast and stromal cells as well as extravillous intermediate trophoblasts (Figure 1, B-E). To compare the APP protein expression level quantitatively, representative trophoblastic areas on all the slides were scanned by the Aperio ScanScope digital pathology system (Leica Biosystems). The APP immunoreactivity in chorionic villi of DS placenta was, on average, approximately 2.3 -fold that of normal placentas $(P<0.0001)$ (Figure $1 \mathrm{~F})$.

\section{Overexpression of APP Suppresses Cell Growth}

To investigate the functional impact of APP overexpression on trophoblast, inducible APP expression was established in the trophoblast cell line HTR-8/SVneo. HTR-8/SVneo was stably transfected with pTRE3G-BI-APP, and single clones were selected. Two clones, B2 and B10, were used for subsequent studies because of their responsiveness to induction by the recombinant inducer protein Tet-Express. Titration of Tet-Express showed that both $\mathrm{B} 2$ and $\mathrm{B} 10$ responded dose dependently by expressing APP at different levels (Figure 2A). When induced, pTRE3G-BI-APP also expresses ZsGreen1 Fluorescent Protein, which serves as an induction marker (Figure 2A). Adding Tet-Express to parental HTR-8/ SVneo had no observable effect on the morphology or cell growth rate (data not shown).

B2 and B10, induced to express APP, grew slightly slower than cells without induction (Figure 2B). This decelerated cell growth was not attributable to lower cell proliferation, because induced cells incorporated $\mathrm{BrdU}$ at the rate as uninduced cells (Figure 2C). There was increased apoptosis, however, in the APP-induced cells, as suggested by increased DNA fragmentation observed by flow cytometry (Figure 2, D and E), increased presence of cleaved caspase 3 (Figure 2F), and increased TUNEL signal (Figure 2G).

To further examine whether APP overexpression is associated with higher apoptotic activity in DS placenta, 


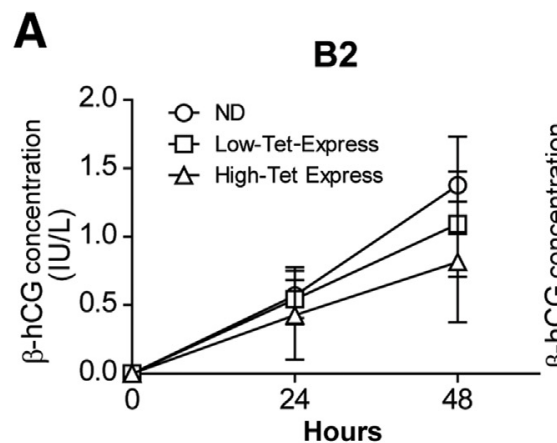

C

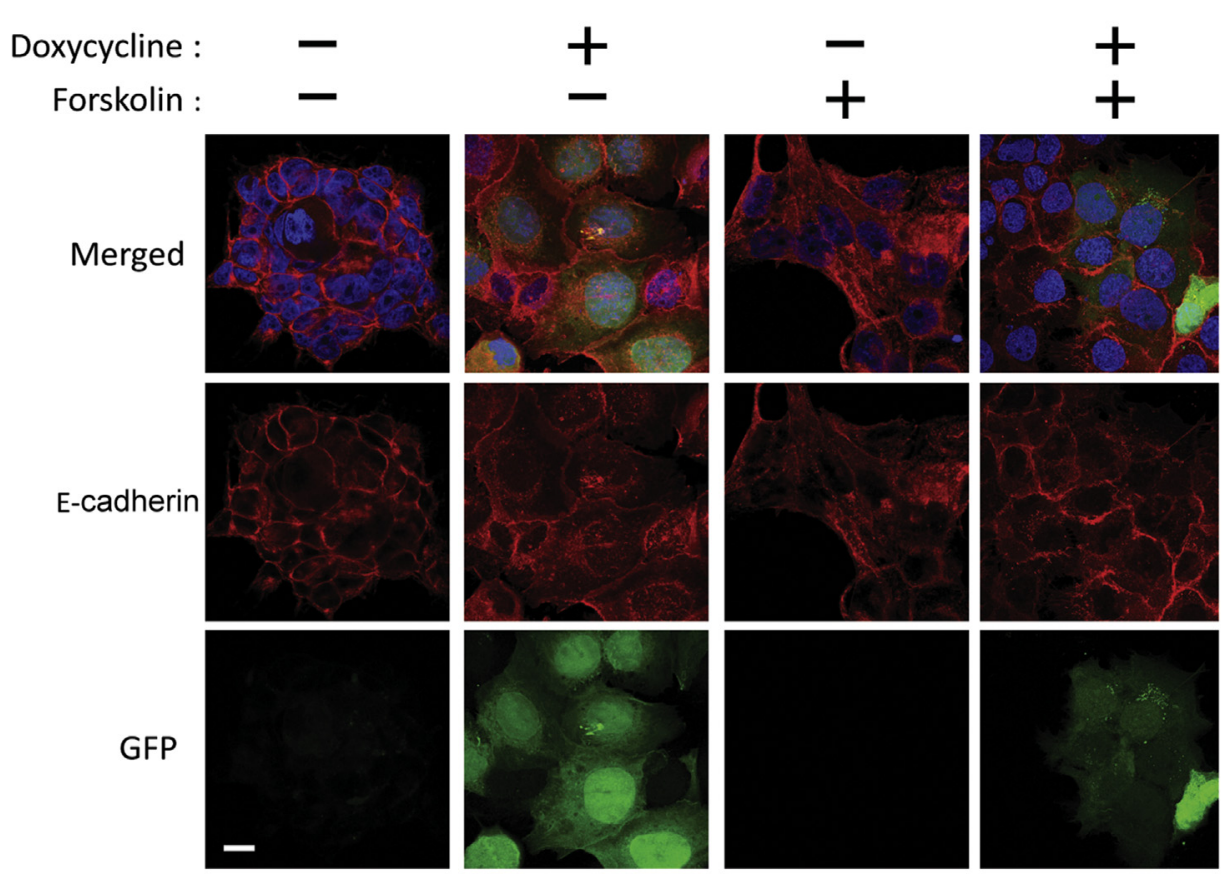

Doxycycline $(\mathrm{ng} / \mathrm{mL}): \quad 0 \quad 10 \quad 100$

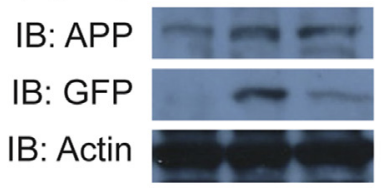

D
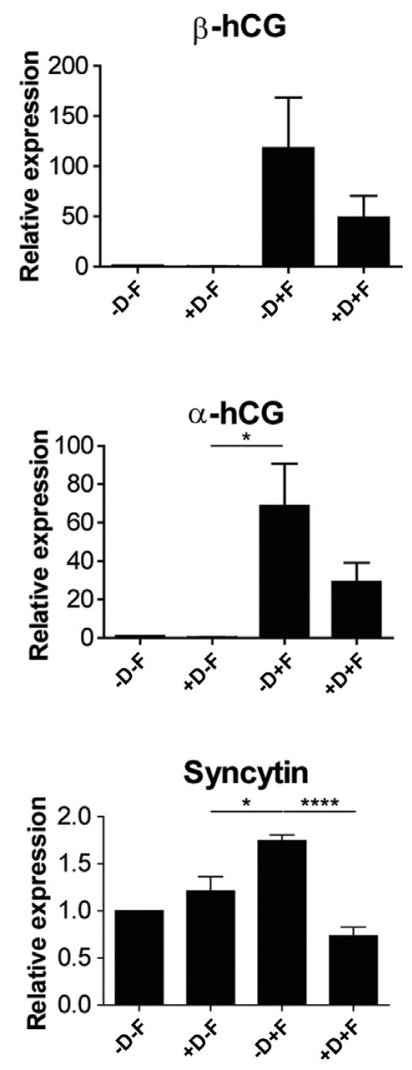

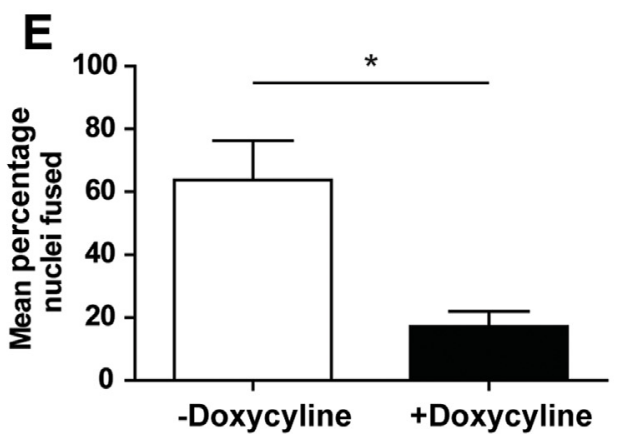

$\mathbf{F}$

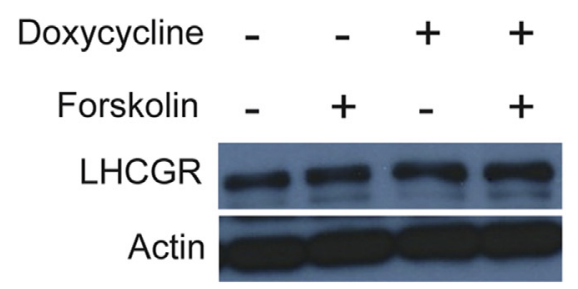

Figure 4 Amyloid precursor protein (APP) overexpression impairs human chorionic gonadotropin (hCG) secretion and syncytialization. A: HTR-8/SVneoAPP was induced to express APP, and the $\beta$-hCG content in the conditioned medium was measured with enzyme-linked immunosorbent assay. B: BeWo-Tet3G was transiently transfected with pTRE3G-BI-APP and induced with indicated concentrations of doxycycline (D). C: BeWo-Tet3G was pretreated with $10 \mathrm{ng} / \mathrm{mL}$ doxycycline or vehicle and then induced to syncytialize by $100 \mu \mathrm{mol} / \mathrm{L}$ forskolin (F) treatment. The cells were observed 24 hours after forskolin treatment. Cell junctions were visualized by E-cadherin immunofluorescence staining. D: Expression of $\alpha$-hCG, $\beta$-hCG, and syncytin transcripts was measured by real-time quantitative RT-PCR. E: The percentage of nuclei belonging to fused cells was counted in forskolin-treated BeWo with or without APP induction. F: The expression of mature hCG receptor (LHCGR) molecules in induced cells was assessed with Western blot. Data are expressed as means \pm SD (A) or means \pm SEM (D and $\mathbf{E}) . n=3$ independent experiments (A and $\mathbf{E}) ; n=4$ independent experiments (D). ${ }^{*} P<0.05, * * * P<0.0001$. Scale bar $=20 \mu \mathrm{m}$ (C). GFP, green fluorescent protein; IB, immunoblot; ND, uninduced. 
TUNEL was performed in FFPE blocks of 10 normal and 10 DS placentas. Although only scanty TUNEL signals could be observed predominantly at the nuclei of the syncytiotrophoblasts of normal placental villi, more frequent TUNEL signals were found in DS placental villi, also prevalent at the nuclei of syncytiotrophoblasts (Figure $2 \mathrm{H}$ ), concurring with the previous findings. ${ }^{24}$

\section{Overexpression of APP Impedes Cell Migration/Invasion}

To evaluate the effect of APP overexpression on cell mobility and invasiveness, B2 and B10 cells were induced to express APP and used in transwell migration/invasion assays. Both clones displayed dose-dependent reduction in the number of cells migrated through the membrane (Figure 3A). A similar trend was observed in Matrigelcoated transwells (invasion assay), which suggested that B2 and B10 migrated and invaded through transwell slower when induced with APP (Figure 3A). Phosphorylation of ERK1/2 is a key event in activating the invasive ability of extravillous trophoblasts. ${ }^{25}$ The phosphorylation status of ERK1/2 was, therefore, investigated in the induced cells. Concurring with the reduced cell mobility and invasiveness, phosphorylation of ERK1/2 was suppressed by APP overexpression (Figure 3B).

\section{Overexpression of APP Interferes with Trophoblast Endocrine Functions}

To examine whether APP overexpression interferes with hCG secretion, the conditioned medium of $\mathrm{B} 2$ and $\mathrm{B} 10$, induced to express APP, was collected, and total $\beta$-hCG concentration was measured. Over a period of 48 hours, the amount of hCG in the conditioned medium of uninduced B2 and B10 accumulated (Figure 4A). APP induction dose dependently reduced the amount of total $\beta$-hCG secreted by the cells (Figure 4A).

Because hCG is mainly produced by syncytiotrophoblasts, we hypothesized that APP overexpression has an impact on the cytotrophoblast-syncytiotrophoblast differentiation. Cultured cytotrophoblasts can be induced to fuse by forskolin treatment. ${ }^{26}$ HTR-8/SVneo, however, could not undergo syncytialization (data not shown). Another inducible system, BeWo-Tet3G, transiently transfected with pTRE3G-BI-APP, induced by doxycycline, was therefore used to assess the effect of APP overexpression on syncytialization (Figure 4B). ${ }^{27}$ Syncytialization was observed through E-cadherin immunofluorescence of BeWo-Tet3G cells. Distinct immunoreactivity for E-cadherin at the cell membrane of individual cells was found in uninduced BeWo-Tet3G cells, highlighting the cell boundaries (Figure 4C). On forskolin treatment, multinucleated cells with no observable E-cadherin separating the nuclei, suggestive of syncytialization, were found (Figure 4C). Induced APP expression (marked by green fluorescent protein) reduced the number of such multinucleated cells (Figure 4, $\mathrm{C}$ and E). Forskolin treatment also led to increased $\alpha$-hCG and $\beta$-hCG, as well as syncytin transcript, as assessed by RT-qPCR, again supportive of syncytialization (Figure 4D). Although APP induction slightly and insignificantly upregulated syncytin, it significantly suppressed forskolinmediated up-regulation of syncytin; however, it did not reach statistical significance for $\alpha$-hCG and $\beta$-hCG (Figure 4D). To quantitate the effect of APP expression, the

Table 1 Top 20 Enriched G0 Terms from Peptides Up-Regulated on APP Overexpression.

\begin{tabular}{|c|c|c|}
\hline G0 term & No. of genes & $P$ value \\
\hline G0:0098609 cell-cell adhesion & 63 & $1.63 \times 10^{-36}$ \\
\hline G0:0006413 translational initiation & 38 & $6.91 \times 10^{-25}$ \\
\hline G0:0006412 translation & 42 & $1.52 \times 10^{-18}$ \\
\hline G0:0006614 SRP-dependent cotranslational protein targeting to membrane & 27 & $3.85 \times 10^{-18}$ \\
\hline G0:0000184 nuclear-transcribed mRNA catabolic process, nonsense-mediated decay & 29 & $2.09 \times 10^{-17}$ \\
\hline G0:0006457 protein folding & 25 & $1.55 \times 10^{-9}$ \\
\hline G0:1904874 positive regulation of telomerase RNA localization to Cajal body & 9 & $4.07 \times 10^{-9}$ \\
\hline G0:1900034 regulation of cellular response to heat & 16 & $7.20 \times 10^{-9}$ \\
\hline G0:0006418 tRNA aminoacylation for protein translation & 12 & $2.18 \times 10^{-8}$ \\
\hline G0:0006397 mRNA processing & 22 & $1.55 \times 10^{-7}$ \\
\hline G0:0017148 negative regulation of translation & 12 & $1.30 \times 10^{-6}$ \\
\hline G0:0016032 viral process & 27 & $2.04 \times 10^{-6}$ \\
\hline G0:0043488 regulation of mRNA stability & 15 & $3.22 \times 10^{-6}$ \\
\hline
\end{tabular}

APP, amyloid precursor protein; ER, endoplasmic reticulum; G0, gene ontology; SRP, signal recognition particle. 
number of fused cells after forskolin treatment with or without APP induction was counted. APP significantly reduced the number of fused cells (Figure 4E). These data support our hypothesis that APP has a negative impact on the fusion and differentiation from cytotrophoblast into syncytiotrophoblast.

Previous studies have reported that mature LHCGR, the receptor for hCG, was significantly reduced in DS compared with normal pregnancies. ${ }^{28}$ It was, therefore, investigated whether APP overexpression affected LHCGR expression in induced BeWo. In this study, when BeWo was induced to syncytialize by forskolin treatment, APP-induced expression did not reduce LHCGR, as demonstrated by Western blot analysis (Figure 4F). Hence, the differentiation inhibition by APP was unlikely to be attributable to reduced LHCGR signaling.

\section{APP Overexpression May Lead to Dysregulation of Cell- Cell Adhesion, Protein Translation, mRNA Processing, and Proteostasis}

Taken together, change was observed in trophoblast functions caused by APP overexpression in trophoblast, including increased cell death, reduced cell mobility and invasiveness, and less efficient syncytialization. To reveal the signaling pathways leading to these phenotypes, the proteomic changes on APP induction in one of the HTR-8/ SVneo clones were characterized. The lysate of uninduced and induced B10 clone was analyzed by liquid chromatography-tandem mass spectrometry. A total of 795 significantly differentially expressed peptides were identified, of which 533 were up-regulated in induced B10. The list of up-regulated proteins was examined using gene ontology (GO) analysis by DAVID to explore the biological processes that the proteins are involved in (Table 1). The most highly enriched proteins were related to cell-cell adhesion functions. This may explain the reduced migratory ability phenotype of induced cells. Moreover, the list contained several GO terms related to protein translation and processing (eg, GO:0006413 translational initiation; GO:0006412 translation; GO:0000184 nuclear-transcribed mRNA catabolic process, nonsense-mediated decay; GO:0000398 mRNA splicing, via spliceosome; and GO:0017148 negative regulation of translation). Furthermore, proteins involved in protein folding were also up-regulated (GO:0006457 protein folding). Because translation regulation represents an important aspect of unfolded protein response, the protein level alterations observed might actually reflect changes in the proteostasis network in response to endoplasmic reticulum stress.

\section{Discussion}

Placental dysfunction is a major cause of growth retardation, a feature commonly found in DS fetuses. However, the role of placental maldevelopment in manifestation of DS phenotypes is unclear, and the mechanism behind this has seldom been studied. APP is the first protein known to be up-regulated in the brain of DS patients. ${ }^{28}$ It has been intensively investigated for its roles in the mental challenge of DS patients, including the early-onset Alzheimer disease commonly found in DS patients. ${ }^{29}$ However, the effect of APP on placental development in DS has never been studied. Herein, solid evidence is provided that APP is overexpressed in the placentas of DS subjects. The up-regulation of mRNA and protein expression was confirmed by RT-qPCR and immunohistochemistry, respectively.

The pathologic impact of APP in DS placentas was investigated by establishing inducible expression systems in two trophoblast models: HTR-8/SVneo and BeWo. Different doses of inducer were selected so that the induced protein expression level in HTR-8/SVneo was comparable to the clinical scenario of trisomy 21 (ie, 1.5 times normal). The first effect of APP overexpression in HTR-8/SVneo observed was a reduction of cell growth caused by increased apoptosis, as evidenced by flow cytometry, TUNEL, and cleaved caspase 3 assays (Figure 2, D-G). APP has been reported to be associated with apoptosis induction in several cell types. For instance, APP overexpression is capable of inducing apoptosis in olfactory neurons through a $\beta$-amyloid-independent mechanism. ${ }^{30}$ Activation of APP by an APP-directed antibody alters the ratio between Tap73 and $\Delta \mathrm{Np} 73$ and favors neuronal apoptosis. ${ }^{31}$ More important, microscopic examination of the maternal-fetal interface of DS conceptuses revealed increased apoptosis at cytotrophoblasts, as demonstrated by pyknotic or fragmented nuclei. ${ }^{32}$ Our results provide the first evidence supporting the involvement of APP in trophoblast cell death.

Furthermore, cytotrophoblast differentiation into syncytiotrophoblast was suppressed on APP overexpression, as evidenced by reduced hCG production and syncytialization, illustrated by reduction in formation of multinucleated cells, syncytin transcript assay, and E-cadherin immunofluorescence (Figure 4). Such phenotypes are reminiscent of the in vitro findings of trophoblasts isolated from DS pregnancies. $^{4,7}$ Therefore, APP overexpression appears to be a major contributor to the reported trophoblast abnormalities in DS pregnancies.

Trophoblasts overexpressing APP migrated and invaded much slower than uninduced cells (Figure 3A). In addition, a particularly high expression of APP at extravillous intermediate trophoblasts of DS placenta was noticed by immunohistochemistry when compared with their counterpart in normal placenta (Figure 1, D and E). However, statistical comparison specifically between extravillous intermediate trophoblasts in DS and normal placentas cannot be conducted because only a small number of tissue sections harbor extravillous trophoblast in our series. Extrapolating such immunohistochemical findings with our in vitro results tends to suggest that DS extravillous trophoblasts would 
function suboptimally regarding their cell motility. It is known that extravillous intermediate trophoblasts are capable of invading the decidua, permeating the smooth muscle layer of the spiral arteries, and replacing the endothelium of the vessels. ${ }^{33}$ Indeed, differentiation of cytotrophoblasts along the invasive pathway has been reported to be impaired in DS pregnancies. ${ }^{32}$ Suppression of APP expression has been reported to promote ovarian cancer cell invasion. ${ }^{34}$ Increasing evidence has also suggested that full-length APP functions as a cell adhesion molecule participating in key events of neuronal development, such as migration, neurite outgrowth, growth cone pathfinding, and synaptogenesis. ${ }^{35}$ APP overexpression induced by in utero electroporating human cDNA resulted in accelerated neuronal migration into the cortex. ${ }^{36}$ APP is also capable of binding to various extracellular matrix molecules, including laminin, collagen, spondin, heparin sulfate, glypican, and Reelin, and thereby regulating neuronal growth cone migration. ${ }^{35}$ The proteomic analysis of APP-induced cells also highlighted proteins involved in cell adhesion as the most significantly enriched GO terms among proteins up-regulated by APP (Table 1). The implication of these proteins on trophoblast mobility and invasiveness will be further investigated.

In summary, these results indicate that dysregulation of trophoblast cell functions on APP induction may play a pivotal role in abnormal placental development in DS. Disruption of the proteostatsis network is likely to be the mediating effect of APP on placental trophoblasts. Further investigations on the mechanisms that underlie this APP-induced dysregulation on trophoblastic functions are in progress.

\section{Acknowledgments}

We thank The University of Hong Kong Li Ka Shing Faculty of Medicine Faculty Proteomics and Metabolomics Core Facility for assistance with liquid chromatography-tandem mass spectrometry; Po Wong and Prof. William Yeung for help on human chorionic gonadotropin measurement; all patients, physicians, and technical and secretarial staff for assistance in this project; and Dr. Peeyush K. Lala for providing HTR-8/SVneo.

\section{References}

1. Antonarakis SE, Lyle R, Dermitzakis ET, Reymond A, Deutsch S: Chromosome 21 and Down syndrome: from genomics to pathophysiology. Nat Rev Genet 2004, 5:725-738

2. Qureshi F, Jacques SM, Johnson MP, Hume RF Jr, Kramer RL, Yaron Y, Evans MI: Trisomy 21 placentas: histopathological and immunohistochemical findings using proliferating cell nuclear antigen. Fetal Diagn Ther 1997, 12:210-215

3. Scifres CM, Nelson DM: Intrauterine growth restriction, human placental development and trophoblast cell death. J Physiol 2009, 587 : 3453-3458

4. Pidoux G, Gerbaud P, Cocquebert M, Segond N, Badet J, Fournier T, Guibourdenche J, Evain-Brion D: Human trophoblast fusion and differentiation: lessons from trisomy 21 placenta. Placenta 2012, 33 Suppl:S81-S86

5. Ricco R, Dalena AM, Valente T, Lettini T, Sanguedolce F, Delfino AR: Quantitative study of placental villi in trisomy by analytical morphometry. Anal Quant Cytol Histol 2009, 31:41-48

6. Roberts L, Sebire NJ, Fowler D, Nicolaides KH: Histomorphological features of chorionic villi at 10-14 weeks of gestation in trisomic and chromosomally normal pregnancies. Placenta 2000, 21: 678-683

7. Pidoux G, Guibourdenche J, Frendo JL, Gerbaud P, Conti M, Luton D, Muller F, Evain-Brion D: Impact of trisomy 21 on human trophoblast behaviour and hormonal function. Placenta 2004, 25 Suppl A: S79-S84

8. Frendo JL, Guibourdenche J, Pidoux G, Vidaud M, Luton D, Giovangrandi Y, Porquet D, Muller F, Evain-Brion D: Trophoblast production of a weakly bioactive human chorionic gonadotropin in trisomy 21-affected pregnancy. J Clin Endocrinol Metab 2004, 89: $727-732$

9. Pidoux G, Gerbaud P, Marpeau O, Guibourdenche J, Ferreira F, Badet J, Evain-Brion D, Frendo JL: Human placental development is impaired by abnormal human chorionic gonadotropin signaling in trisomy 21 pregnancies. Endocrinology 2007, 148: 5403-5413

10. Lana-Elola E, Watson-Scales SD, Fisher EM, Tybulewicz VL: Down syndrome: searching for the genetic culprits. Dis Model Mech 2011, 4 $586-595$

11. Rozovski U, Jonish-Grossman A, Bar-Shira A, Ochshorn Y, Goldstein M, Yaron Y: Genome-wide expression analysis of cultured trophoblast with trisomy 21 karyotype. Hum Reprod 2007, 22: $2538-2545$

12. Neve RL, Finch EA, Dawes LR: Expression of the Alzheimer amyloid precursor gene transcripts in the human brain. Neuron 1988, 1: 669-677

13. Weksler ME, Szabo P, Relkin NR, Reidenberg MM, Weksler BB, Coppus AM: Alzheimer's disease and Down's syndrome: treating two paths to dementia. Autoimmun Rev 2013, 12:670-673

14. Moncaster JA, Pineda R, Moir RD, Lu S, Burton MA, Ghosh JG, Ericsson M, Soscia SJ, Mocofanescu A, Folkerth RD, Robb RM, Kuszak JR, Clark JI, Tanzi RE, Hunter DG, Goldstein LE: Alzheimer's disease amyloid-beta links lens and brain pathology in Down syndrome. PLoS One 2010, 5:e10659

15. Pallister C, Jung SS, Shaw I, Nalbantoglu J, Gauthier S, Cashman NR: Lymphocyte content of amyloid precursor protein is increased in Down's syndrome and aging. Neurobiol Aging 1997, 18:97-103

16. Govoni S, Bergamaschi S, Gasparini L, Quaglia C, Racchi M, Cattaneo E, Binetti G, Bianchetti A, Giovetti F, Battaini F, Trabuechi M: Fibroblasts of patients affected by Down's syndrome oversecrete amyloid precursor protein and are hyporesponsive to protein kinase C stimulation. Neurology 1996, 47:1069-1075

17. Graham CH, Hawley TS, Hawley RG, MacDougall JR, Kerbel RS, Khoo N, Lala PK: Establishment and characterization of first trimester human trophoblast cells with extended lifespan. Exp Cell Res 1993, 206:204-211

18. Haynes HR, Killick-Cole CL, Hares KM, Redondo J, Kemp KC, Moutasim KA, Faulkner C, Wilkins A, Kurian KM: Evaluation of the quality of RNA extracted from archival FFPE glioblastoma and epilepsy surgical samples for gene expression assays. J Clin Pathol 2018, 71:695-701

19. Siu MK, Wong ES, Chan HY, Ngan HY, Chan KY, Cheung AN Overexpression of NANOG in gestational trophoblastic diseases: effect on apoptosis, cell invasion, and clinical outcome. Am J Pathol 2008, $173: 1165-1172$

20. Mak VC, Wong OG, Siu MK, Wong ES, Ng WY, Wong RW, Chan KK, Ngan HY, Cheung AN: FBI-1 is overexpressed in gestational trophoblastic disease and promotes tumor growth and cell aggressiveness of choriocarcinoma via PI3K/Akt signaling. Am J Pathol 2015, 185:2038-2048 
21. Wisniewski JR, Zougman A, Nagaraj N, Mann M: Universal sample preparation method for proteome analysis. Nat Methods 2009, 6: 359-362

22. Meller M, Vadachkoria S, Luthy DA, Williams MA: Evaluation of housekeeping genes in placental comparative expression studies. Placenta 2005, 26:601-607

23. Kaitu'u-Lino TJ, Hastie R, Cannon P, Lee S, Stock O, Hannan NJ, Hiscock R, Tong S: Stability of absolute copy number of housekeeping genes in preeclamptic and normal placentas, as measured by digital PCR. Placenta 2014, 35:1106-1109

24. Wong SY, Ngan HY, Chan CC, Cheung AN: Apoptosis in gestational trophoblastic disease is correlated with clinical outcome and Bcl-2 expression but not Bax expression. Mod Pathol 1999, 12:1025-1033

25. Gupta SK, Malhotra SS, Malik A, Verma S, Chaudhary P: Cell signaling pathways involved during invasion and syncytialization of trophoblast cells. Am J Reprod Immunol 2016, 75:361-371

26. Wice B, Menton D, Geuze H, Schwartz AL: Modulators of cyclic AMP metabolism induce syncytiotrophoblast formation in vitro. Exp Cell Res 1990, 186:306-316

27. Yoshie M, Kashima H, Bessho T, Takeichi M, Isaka K, Tamura K: Expression of stathmin, a microtubule regulatory protein, is associated with the migration and differentiation of cultured early trophoblasts. Hum Reprod 2008, 23:2766-2774

28. Banerjee S, Smallwood A, Chambers AE, Papageorghiou A, Loosfelt H, Spencer K, Campbell S, Nicolaides K: A link between high serum levels of human chorionic gonadotrophin and chorionic expression of its mature functional receptor (LHCGR) in Down's syndrome pregnancies. Reprod Biol Endocrinol 2005, 3:25
29. Castro P, Zaman S, Holland A: Alzheimer's disease in people with Down's syndrome: the prospects for and the challenges of developing preventative treatments. J Neurol 2017, 264:804-813

30. Cheng N, Jiao S, Gumaste A, Bai L, Belluscio L: APP overexpression causes Abeta-independent neuronal death through intrinsic apoptosis pathway. eNeuro 2016, 3:ENEURO.0150-16.2016

31. Benosman S, Meng X, Von Grabowiecki Y, Palamiuc L, Hritcu L, Gross I, Mellitzer G, Taya Y, Loeffler JP, Gaiddon C: Complex regulation of p73 isoforms after alteration of amyloid precursor polypeptide (APP) function and DNA damage in neurons. J Biol Chem 2011, 286:43013-43025

32. Wright A, Zhou Y, Weier JF, Caceres E, Kapidzic M, Tabata T, Kahn M, Nash C, Fisher SJ: Trisomy 21 is associated with variable defects in cytotrophoblast differentiation along the invasive pathway. Am J Med Genet A 2004, 130A:354-364

33. Lunghi L, Ferretti ME, Medici S, Biondi C, Vesce F: Control of human trophoblast function. Reprod Biol Endocrinol 2007, 5:6

34. Fan X, Liu Y, Jiang J, Ma Z, Wu H, Liu T, Liu M, Li X, Tang H: miR20a promotes proliferation and invasion by targeting APP in human ovarian cancer cells. Acta Biochim Biophys Sin 2010, 42:318-324

35. Sosa LJ, Caceres A, Dupraz S, Oksdath M, Quiroga S, Lorenzo A: The physiological role of the amyloid precursor protein as an adhesion molecule in the developing nervous system. J Neurochem 2017, 143: $11-29$

36. Young-Pearse TL, Bai J, Chang R, Zheng JB, LoTurco JJ, Selkoe DJ: A critical function for beta-amyloid precursor protein in neuronal migration revealed by in utero RNA interference. J Neurosci 2007, 27: 14459-14469 